\title{
Robotic-Assisted Partial Nephrectomy: Surgical Technique Using a 3-Arm Approach and Sliding-clip Renorrhaphy
}

\author{
Jose M. Cabello, Brian M. Benway, Sam B. Bhayani \\ Department of Surgery, Division of Urologic Surgery, Washington University School of Medicine, \\ Saint Louis, Missouri, USA
}

\begin{abstract}
Introduction: For the treatment of renal tumors, minimally invasive nephron-sparing surgery has become increasingly performed due to proven efficiency and excellent functional and oncological outcomes. The introduction of robotics into urologic laparoscopic surgery has allowed surgeons to perform challenging procedures in a reliable and reproducible manner. We present our surgical technique for robotic assisted partial nephrectomy (RPN) using a 3-arm approach, including a sliding-clip renorrhaphy.

Materials and Methods: Our RPN technique is presented which describes the trocar positioning, hilar dissection, tumor identification using intraoperative ultrasound for margin determination, selective vascular clamping, tumor resection, and reconstruction using a sliding-clip technique.

Conclusion: RPN using a sliding-clip renorrhaphy is a valid and reproducible surgical technique that reduces the challenge of the procedure by taking advantage of the enhanced visualization and control afforded by the robot. The renorrhaphy described is performed under complete control of the console surgeon, and has demonstrated a reduction in the warm ischemia times in our series.
\end{abstract}

Key words: kidney; nephrectomy; surgical procedures, minimally invasive; robotics Int Braz J Urol. 2009; 35: 199-204

\section{INTRODUCTION}

The diagnosis and treatment of renal tumors has evolved over the last 15 years, spurred by the increased incidental detection of small, asymptomatic renal masses on abdominal imaging, as well as by the widespread adoption of nephron-sparing surgical techniques. Even for patients with normal renal function, nephron-sparing techniques have been advocated whenever feasible due to the low morbidity and excellent long-term functional and oncological outcomes (1).

The constant search for solutions that combine optimal outcomes with less inconvenience for the patients, better cosmetic results, and shorter hospitalization has lead to the validation of laparoscopic partial nephrectomy (LPN) as a viable and acceptable alternative to traditional open partial nephrectomy (OPN) (1). However, the major obstacle to the widespread use of LPN is its technical difficulty and steep learning curve, particularly with regards to renal reconstruction and intracorporeal knot tying, which are generally performed under the time constraints of warm ischemia.

The introduction of robotics into urologic laparoscopic surgery has provided remarkable enhancements to minimally-invasive procedures by adding three-dimensional visualization, wrist-emu- 
lated mobility for the instruments, better ergonomics for the surgeon, and the reduction of tremor. Several reported series have described various techniques for robotic partial nephrectomy, but commonly lack an appropriate description of an efficient, effective, and reproducible technique for renorrhaphy (2-3).

Sliding-clip renorrhaphy, a technique for renal reconstruction, was recently refined at our institution, and described in a prior report (4). Since its introduction in 2007 and display at the 2008 Worldwide Robotic Renal Symposium, it has been gaining acceptance due to its ease of implementation and quality of repair.

The objective of this report is to describe our technique for robotic-assisted partial nephrectomy (RPN) using a 3-arm approach, including the slidingclip renorrhaphy technique.

\section{SURGICAL TECHNIQUE}

Under general anesthesia, the patient is placed in a flexed, full flank position. A Veress needle is used to establish a pneumoperitoneum of $15 \mathrm{~mm}$ $\mathrm{Hg}$. A $12 \mathrm{~mm}$ camera port is placed approximately 2 to $5 \mathrm{~cm}$ superior to the umbilicus. Under direct vision, the $8 \mathrm{~mm}$ robotic trocars are placed, one subcostal and one in the lower quadrant. This provides appropriate triangulation of the instruments. A 12 $\mathrm{mm}$ assistant port is placed in between the robotic trocar sites, cephalad or caudal depending on the tumor location, and at least $1 \mathrm{~cm}$ below the plane of the camera port. For right-sided masses, a $5 \mathrm{~mm}$ subxiphoid trocar can be placed for liver retraction if necessary (Figure-1). The robot is then docked at an angle, centered along the line defined by the camera port and the renal hilum.

For this approach, a 30-degree down lens is used. The robotic instruments used include monopolar scissors, Prograsp forceps, and robotic needle drivers. The assistant's responsibilities include retraction and clearing of the field with a laparoscopic suction device, as well as placing bulldog clamps for hilar control, and passing Weck (Teleflex, Research Triangle Park, NC) Hem-o-Lock clips and LapraTy (Ethicon, Cincinnati, $\mathrm{OH}$ ) clips utilized during the renorrhaphy.
The kidney is exposed by incising the peritoneum sharply along the white line of Toldt and reflecting the colon medially to provide optimal exposure of the retroperitoneal space (Figure-2). Next, the ureter, gonadal vessels, and the lower pole of the kidney are identified and retracted laterally, placing the renal vessels on stretch, a maneuver that aids the hilar dissection. The identification of the hilar structures is performed using standard laparoscopic techniques. The isolation of renal artery and vein is necessary for selective vascular clamping, and is achieved by gently pushing the fat off both the front and the back of the vessels (Figure-3).

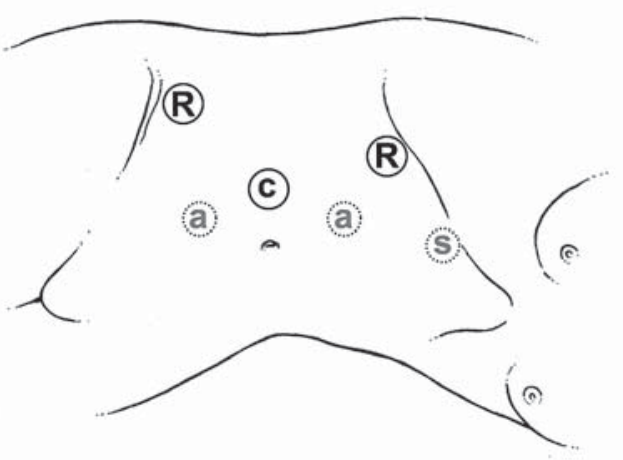

Figure 1 - Trocar positioning for the 3-arm approach. $C=$ Camera port $(12 \mathrm{~mm}), R=$ robotic arms $(8 \mathrm{~mm}), a=$ alternatives for assistant trocar $(12 \mathrm{~mm}), \mathrm{s}=$ additional port for liver retractor $(5 \mathrm{~mm})$.

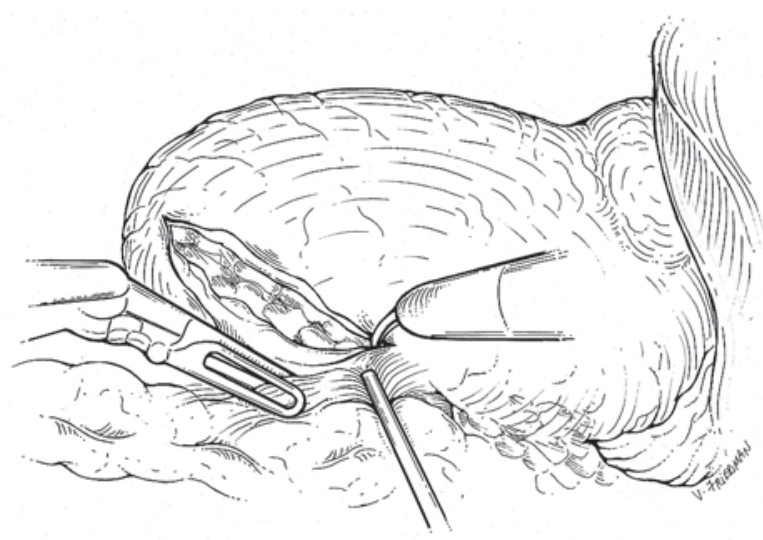

Figure 2 - The kidney is exposed by reflecting the colon medially. 


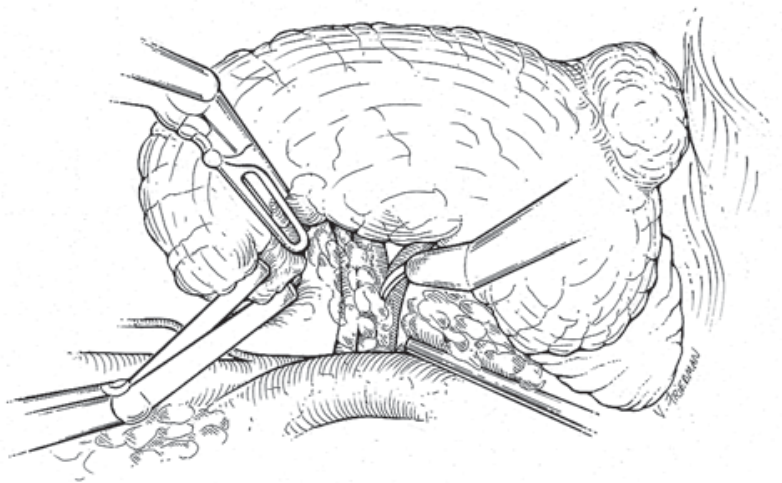

Figure 3 - The kidney is retracted laterally to allow adequate exposure and aid hilar dissection.

Next, attention is focused on tumor localization. If necessary, the kidney can be mobilized for upper pole or posterior tumors. For both endophytic and exophytic tumors, an ultrasound is performed to define the extent of the mass. The fat overlying the normal parenchyma is dissected free; however, the fat overlying the mass is left intact for pathologic analysis. The capsule of the kidney is scored with cautery (Figure-4).

Vascular clamping is performed with bulldog clamps placed by the assistant after mannitol (12.5 or $25 \mathrm{gm}$ ) infusion. In our technique we prefer arterial and venous clamping separately, ensuring a bloodless

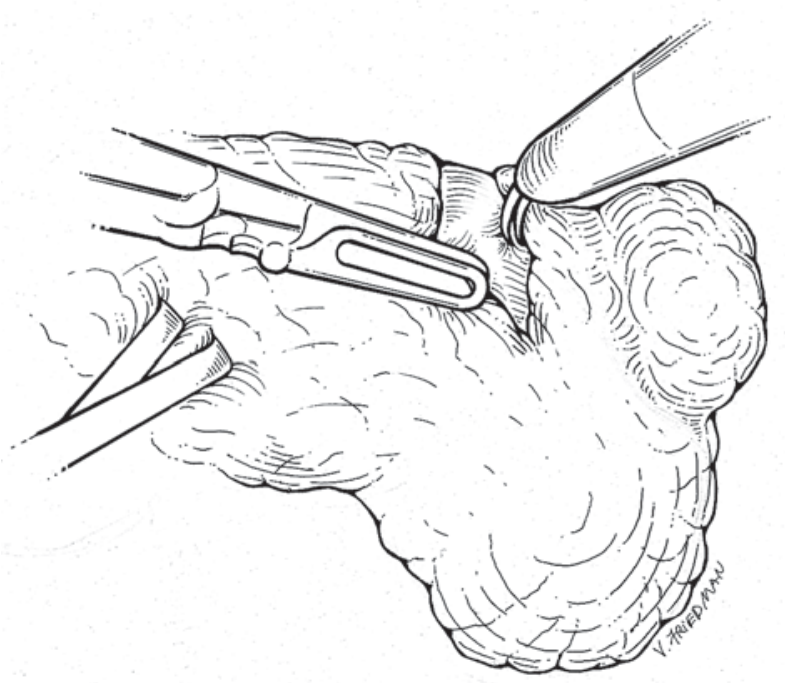

Figure 4 - Renal mass identification and determination of margins of resection. field during the resection (Figure-5). Renal ischemia is confirmed by evaluating the change in color, size, and consistency of the kidney.

Tumor excision is then performed following standard oncological principles, preferentially with monopolar scissors and the aid of the assistant's suction for providing countertraction and maintaining a dry field. Once complete, the specimen is placed beside the kidney or on the top of the liver for later retrieval. Biopsy samples are obtained by the assistant from the surgical bed and sent for frozen section analysis.

For the reconstruction phase, the scissor is exchanged for a needle driver, and the Prograsp is retained for use in tightening sutures. Adequate hemostasis is then achieved using cautery and, in some cases, placing figure-of-eight sutures to address larger veins or arteries. If the collecting system is entered during the excision, it is oversewn with 2-0 polyglactin on an SH needle, and a LapraTy clip may be used to secure the suture. In select cases, we use a bolster of thrombogenic material that is placed to fill the renal defect.

The renorrhaphy is performed using a slidingclip technique, which is described in detail elsewhere (4). Zero or No. 1 polyglactin sutures on a CT needle are prepared on the back table by cutting to a length of $15 \mathrm{~cm}$, and tying a knot at the end. A LapraTy clip

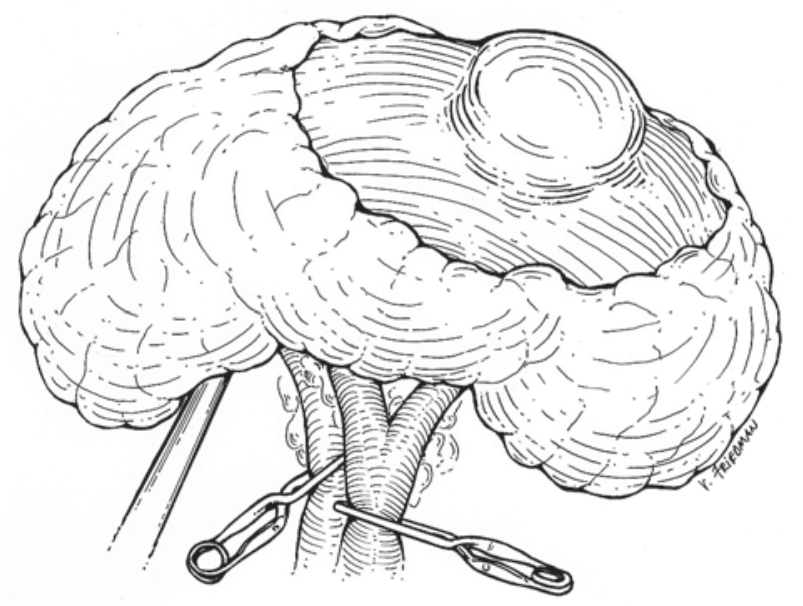

Figure 5 - Selective vascular clamping with bulldogs prior to resection. 
is placed above the knot, followed by a Weck Hemo-Lock clip. These sutures are then placed through the margins of the capsule at intervals of $1 \mathrm{~cm}$. After the final throws have been completed, the assistant places a Hem-o-Lock clip on the loose end of the suture. The surgeon seated at the console is then able to slide the clip towards the repair zone to tighten the renorrhaphy under precise control, allowing for a secure, hemostatic closure. In addition, the tension of the repair can be re-adjusted as necessary. When the optimal closure has been achieved, a LapraTy clip is applied to lock the sliding clip in position (Figure$6)$.

The hilum is then unclamped, starting with the vein, and then the artery. If bleeding occurs after unclamping, it is initially observed as a re-expansion of the perfused kidney which may lead to further tension on the repair, resulting in tamponade. If bleeding persists, the clips may be re-tightened or additional sutures may be added. The use of hemostatic sealant agents is optional, and depends upon the surgeon's preference and size of the defect.

The specimen is then placed in a retrieval bag for extraction using one of the trocar sites. The perirenal fat is repositioned over the kidney and usually secured by placing a Hem-o-Lock clip. If there are concerns over collecting system injury, a closed suction drain may be placed, but is not necessary in all circumstances. Finally, wounds are closed with subcuticular sutures.

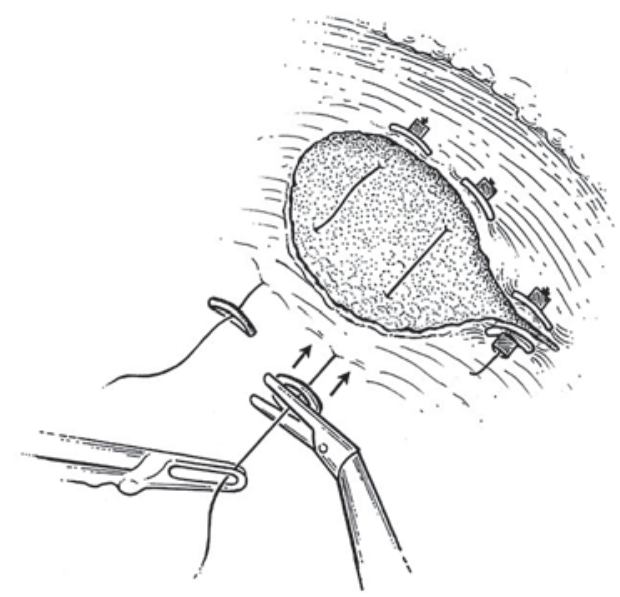

Figure 6 - Sliding-clip renorrhaphy.

\section{COMMENTS}

Minimally invasive nephron-sparing techniques, which have been developed for the treatment of renal tumors, are based on the gold standard of open partial nephrectomy. The surgical principles for identification, dissection, vascular clamping, and resection are essentially the same in all approaches. However, renal reconstruction is more difficult for pure laparoscopic techniques, owing to the confined space, restrictive working angles, limitations of the instruments, two-dimensional view, and reduced tactile awareness, all of which are further exacerbated by the need to limit warm ischemia times.

The use of robotics in laparoscopic surgery aims to solve many of these problems. As previously described, the enhanced stereoscopic visualization and instrument mobility can reduce many of the challenges common to the laparoscopic approach to a considerable degree. Nevertheless, the majority of the techniques for renorrhaphy described in the literature for LPN and RPN are similar, and mimic those used in OPN.

The renorrhaphy technique employed at our institution is a simple, reliable, and reproducible way to achieve an optimal surgeon-controlled reconstruction, which takes advantage of the precision offered by the robotic instruments. The sliding-clip technique provides an efficient and effective closure, which obviates the need for intracorporeal knot tying, and offers the ability to re-tighten the sutures if necessary to ensure proper tension. This technique has been used in the majority of our RPN and has been adopted as the standard renal reconstruction by all surgeons involved in our institutional robotic renal surgery program. Further implementation and its routine use has demonstrated a decrease in the warm ischemia time in comparison with our series of LPN (25 min for LPN v/s 19 min for RPN) (5); and also a similar trend of decrease can be observed when we compare the now standardized procedure with our initial experience of 13 RPN, when the traditional knot-tying and clip closure was used for reconstruction (24 min. for traditional v/s $16 \mathrm{~min}$. for sliding-clip renorrhaphy). However, a focused prospective analysis will be necessary to confirm if this trend is significant. A more detailed description 
of our series and outcomes can be found in prior publications of our group $(5,6)$.

$\mathrm{RPN}$ is a procedure that continues to evolve. Future improvements to the technique and technology are likely to address issues of exposure, vascular control and reconstruction. Many of these innovations are likely to arrive with the introduction of new instruments and combination with ablation techniques in development, which will further reduce the challenge of the procedure, allow for less reliance upon the assistant, and minimize warm ischemic times.

\section{CONFLICT OF INTEREST}

Dr. Sam B. Bhayani is a paid consultant of Intuitive Surgical, Sunnyvale, CA, USA.

\section{REFERENCES}

1. Haber GP, Gill IS: Laparoscopic partial nephrectomy: contemporary technique and outcomes. Eur Urol. 2006; 49: 660-5.

\author{
Correspondence address: \\ Dr. Jose M. Cabello \\ 660 S. Euclid Ave \\ Campus, Box 8242 \\ Saint Louis, MO 63110, USA \\ Fax +1 314 454-5244 \\ E-mail: cabelloj@wudosis.wustl.edu
}

2. Kaul S, Laungani R, Sarle R, Stricker H, Peabody J, Littleton R, Menon M: da Vinci-assisted robotic partial nephrectomy: technique and results at a mean of 15 months of follow-up. Eur Urol. 2007; 51: 186-91; discussion 191-2.

3. Rogers CG, Singh A, Blatt AM, Linehan WM, Pinto PA: Robotic partial nephrectomy for complex renal tumors: surgical technique. Eur Urol. 2008; 53: 51421.

4. Bhayani SB, Figenshau RS: The Washington University Renorrhaphy for robotic partial nephrectomy: a detailed description of the technique displayed at the 2008 World Robotic Urologic Symposium. J Robotic Surg. 2008; 2: 139-40.

5. Wang AJ, Bhayani SB: Robotic Partial Nephrectomy Versus Laparoscopic Partial Nephrectomy for Renal Cell Carcinoma: Single-Surgeon Analysis of $>100$ Consecutive Procedures. Urology. 2008. [Epub ahead of print]

6. Bhayani SB, Das N: Robotic assisted laparoscopic partial nephrectomy for suspected renal cell carcinoma: retrospective review of surgical outcomes of 35 cases. BMC Surg. 2008; 8: 16.

\section{EDITORIAL COMMENT}

Partial nephrectomy for renal tumors smaller than $4 \mathrm{~cm}$ of diameter is currently recommended with similar oncological long term outcomes of radical nephrectomy. However, it remains to be a challenging surgery for both conventional and laparoscopic methods, considering the preservation of the remaining parenchyma, intra and post-operative bleeding and post-operative urinary leakage.
This technical proposal for partial nephrectomy seems very promising and I had the opportunity to testify how elegant one sliding-clip renorrhaphy surgery was in a Robotic Brazilian Symposium in Sao Paulo a few months ago. The bleeding was minimal and the warm ischemia lasted for 20 minutes. However, some remarks should be made. The stitches were used in the same fashion as in pure laparoscopic procedure. 
The only difference is the absence of tying knots. Intra-corporeal knot tying has become easier and faster with DaVinci robot. Therefore, why spend more money using Hem-o-Lok as well as LapraTy clip in sliding-clip technique for renorrhaphy? Using robot the time for knotting should not be so different from the time elapsed for applying these clips. I believe that maybe it is an alternative for cases when the estimated

\section{REPLY BY THE AUTHORS}

We sincerely appreciate Dr. Mitre's thoughtful comments, as he raises some very valid questions regarding our preference for sliding-clip renorrhaphy over a more traditional tied-suture reconstruction. We certainly agree that robot assistance significantly reduces the challenge of intracorporeal knot tying; however, due to the need for delicate handling of the renal tissue, coupled with the need to limit warm ischemic times, tied-suture renorrhaphy remains a relatively challenging technique in this setting.

During a tied-suture closure, the sutures are necessarily pulled at angles off the perpendicular, placing shearing forces upon the capsule, which may lead to inadvertent tearing, even with the use of pledgets. With sliding-clip renorrhaphy, all closing tension is applied perpendicular to the capsule, which we feel reduces the risk of capsular disruption. Moreover, the large footprint of the Hem-O-Lock clip distributes the tension evenly across a comparatively wider surface area, further minimizing the risk of capsular tear.

Furthermore, should the tension on the repair be found to be suboptimal, or if bleeding is encountered after the clamp is removed, sliding-clip renorrhaphy allows the surgeon to adjust the tension of the repair without the need for the placement of additional sutures, something which is not possible using a traditional tied-suture repair.

With regards to the expediency of slidingclip renorrhaphy, we have recently reported our data warm ischemia time might be higher than 30 minutes and perhaps more useful for pure laparoscopic partial nephrectomy especially for those surgeons with low familiarity in reconstructive laparoscopic surgery. Prior to be considered superior than intra-corporeal knot tying reconstruction, the results of both methods must be compared in a prospective analysis.

Dr. Anuar Ibrahim Mitre
Associate Professor of Urology
University Sao Paulo, USP
Sao Paulo, SP, Brazil
E-mail: anuar@mitre.com.br

demonstrating that the implementation of the sliding-clip technique for renorrhaphy is associated with significant reductions of both overall operative times, as well as warm ischemic times, with the latter being reduced by nearly 8 minutes (1).

Although a warm ischemic time of less than 30 minutes is generally considered safe in patients with normal preoperative renal function, the role of robot-assisted partial nephrectomy is ever expanding to include patients with increasing degrees of renal impairment and larger tumors. As such, any technique which may result in a reduction of warm ischemic times may prove critical to the continued success of the robotic approach.

Therefore, we believe that the disadvantages of a slightly higher cost are more than offset by the potential benefits to the patient in terms of maximal preservation of renal reserve. We however, agree that prospective analysis will be needed to further evaluate the utility of sliding-clip renorrhaphy.

\section{REFERENCE}

1. Benway BM, Wang AJ, Cabello JM, Bhayani SB: Robotic partial nephrectomy with sliding-clip renorrhapy: technique and outcomes. Eur Urol. 2009; 55: 592-599.

The Authors 